\title{
Influence of the solar EUV flux on the Martian plasma environment
}

\author{
R. Modolo ${ }^{1}$, G. M. Chanteur ${ }^{1}$, E. Dubinin ${ }^{2}$, and A. P. Matthews ${ }^{3}$ \\ ${ }^{1}$ CETP-IPSL, 10-12 avenue de l'Europe, 78140 Vélizy, France \\ ${ }^{2}$ Max-Planck Institute für Aeronomie, 37191 Katlenburg-Lindau, Germany \\ ${ }^{3}$ School of Pure and Applied Physics, University of KwaZulu-Natal, Durban 4041, South Africa
}

Received: 11 September 2004 - Revised: 22 November 2004 - Accepted: 25 November 2004 - Published: 28 February 2005

\begin{abstract}
The interaction of the solar wind with the Martian atmosphere and ionosphere is investigated by using threedimensional, global and multi-species hybrid simulations. In the present work we focus on the influence of the solar EUV flux on the Martian plasma environment by comparing simulations done for conditions representative of the extrema of the solar cycle. The dynamics of four ionic species $\left(\mathrm{H}^{+}, \mathrm{He}^{++}, \mathrm{O}^{+}, \mathrm{O}_{2}^{+}\right)$, originating either from the solar wind or from the planetary plasma, is treated fully kinetically in the simulation model in order to characterize the distribution of each component of the plasma, both at solar maximum and at solar minimum. The solar EUV flux controls the ionization frequencies of the exospheric species, atomic hydrogen and oxygen, as well as the density, the temperature, and thus the extension of the exosphere. Ionization by photons and by electron impacts, and the main charge exchange reactions are self-consistently included in the simulation model. Simulation results are in reasonable agreement with the observations made by Phobos-2 and Mars Global Surveyor (MGS) spacecraft: 1) the interaction creates a cavity, void of solar wind ions $\left(\mathrm{H}^{+}, \mathrm{He}^{++}\right)$, which depends weakly upon the phase of the solar cycle, 2) the motional electric field of the solar wind flow creates strong asymmetries in the Martian environment, 3) the spatial distribution of the different components of the planetary plasma depends strongly upon the phase of the solar cycle. The fluxes of the escaping planetary ions are computed from the simulated data and results for solar maximum are compared with estimates based on the measurements made by experiments ASPERA and TAUS on board Phobos-2.
\end{abstract}

Key words. Magnetospheric physics (Solar wind and interactions with unmagnetized bodies) - Space plasma physics (Ionization processes; Numerical simulation suites)

\section{Introduction}

Mars does not possess a significant dynamo magnetic field to protect its atmosphere from the solar wind (Acuña et al.,

Correspondence to: R. Modolo

(ronan.modolo@cetp.ipsl.fr)
1998). The deflection of the solar wind around Mars, downstream of the bow shock (BS), results from its interaction with the top atmosphere and the conducting dayside ionosphere; this interaction is similar to the interaction with Venus but presents distinctive features due to the smaller size of planet Mars. The solar wind plasma interacting directly with the Martian upper atmosphere contributes, besides photoionization, to the production of planetary ions by processes such as electron impacts or charge exchanges. The present understanding of this interaction has been recently reviewed by Mazelle et al. (2004) and Nagy et al. (2004). The interaction does not only affect the solar wind flow, but also provides scavenging mechanisms enhancing the loss of planetary matter $(\mathrm{H}, \mathrm{He}, \mathrm{C}, \mathrm{N}$ and $\mathrm{O})$ and thus contributes to the dehydration of Mars over cosmological time scales. The loss of the Martian atmosphere is still not completely understood. This is essentially due to the lack of ion measurements at altitudes less than one thousand kilometers before the Mars Expess mission. Although MAG-ER experiment on board the Mars Global Surveyor provides full observations on the electron population (Acuña et al., 2001), it is not sufficient to characterize the escape mecanism. Phobos-2 spacecraft made observations for different ions species but on ellipticals orbits down to $800 \mathrm{~km}$. Presently, the ASPERA-3 experiment on the Mars-Express spacecraft characterizes precisely ions species at low energies and altitudes, but unfortunately without any information on the magnetic field due to the absence of a magnetometer on board the Mars Express spacecraft. The first results have been published recently (Lundin et al., 2004). Therefore several numerical simulations have been dedicated in the last years to study this particular interaction between the solar wind and the Martian environment. These studies have been carried out using different approaches like gas dynamics models (Spreiter and Stahara, 1992), MHD models (Tanaka, 1993; Liu et al., 1999; Liu et al., 2001; Ma et al., 2002; Ma et al., 2004), and a bi-ion MHD model (Sauer et al., 1994). Mass loading of the solar wind by planetary ions can partly be taken into account by gas dynamics and single fluid MHD models, but kinetics effects due to finite ionic gyroradii remain beyond the capabilities of these models. Multifluid MHD models have partly this capability if they include at least density and momentum equations 
for each species, otherwise responses of the various ionic species to the electromagnetic field are not properly separated. Hybrid models (Brecht and Ferrante, 1993; Shimazu, 1999; Kallio and Janhunen, 2001) retain self-consistently all kinetic effects in the dynamic of the ions.

Mars presents to the solar wind a much smaller obstacle than Venus does. The convective Larmor radius of solar wind ions is not small compared to the planetary radius, and is of the order of the standoff distance of the bow shock to the planet: thus kinetic effects are much more important for Mars than for Venus. Therefore, we adopt a hybrid description where ions are treated kinetically and electrons as a massless fluid. This kind of description allows one to study the behaviour of several ions species in order to compare simulation results with spacecraft observations.

Bruno et al. (1994) have investigated the variations of solar wind parameters over solar cycle numbers 20 and 21, i.e. for years 1966 to 1989, separating slow and fast solar wind. The solar cycle modulates the solar wind density, velocity and proton temperature, as well as the interplanetary magnetic field. It appears that long-term variations of the yearly averaged parameters reported by Bruno et al. (1994) are usually smaller than short-term variations of the hourly averaged parameters: see, for example solar wind data made available through the NSSDC (http://nssdc.gsfc.nasa.gov/). Thus, in order to disentangle the effects of the modulations of the solar wind and radiation, we focus this study on the response of the Martian plasma environment to the variation of the EUV solar flux and the concomitant variation of the Martian neutral environment for average solar wind parameters characteristic of a slow solar wind. In this paper, the maximum (minimum) of the solar EUV flux will simply be refered to as solar maximum (minimum), for convenience. The influence of the varying solar wind parameters for a given EUV flux will be reported in a forthcoming study.

The paper is organized as follows. The simulation model is outlined in Sect. 2, then the simulated Martian plasma environment is presented for solar maximum and for solar minimum in Sect. 3. Fluxes of escaping planetary ions computed from the simulated data are presented in Sect. 4. Results from the simulations for conditions of solar maximum are compared to relevant observations by Phobos- 2 and Mars Global Surveyor spacecraft. Results concerning the simulated magnetic field will be detailed in a future publication.

\section{Simulation model}

As mentioned in the Introduction, the ion gyroradii being comparable to the scales of the plasma flow around Mars, a fully kinetic description of the ions is appropriate. A fully kinetic treatment of the electrons, although desirable to take into account phenomena involving electronic scales, is presently not viable in global simulations of planetary objects. Most of the simulation models used in this field include electrons in the simplest way, retaining only their contribution to charge neutrality and electric currents due to their high mobility.

\subsection{Hybrid model}

In hybrid models ionic species are described by sets of macroparticles: meanwhile, electrons are described by a fluid. The equations of motion of macroparticles are formally identical to the ones for physical particles:

$$
\begin{aligned}
\frac{d \boldsymbol{v}_{\boldsymbol{i}}}{d t} & =\frac{q_{i}}{m_{i}}\left(\boldsymbol{E}+\boldsymbol{v}_{\boldsymbol{i}} \times \boldsymbol{B}\right) \\
\frac{d \boldsymbol{x}_{\boldsymbol{i}}}{d t} & =\boldsymbol{v}_{\boldsymbol{i}},
\end{aligned}
$$

where $\boldsymbol{x}_{\boldsymbol{i}}, \boldsymbol{v}_{\boldsymbol{i}}, q_{i}$ and $m_{\boldsymbol{i}}$ are, respectively, the position, velocity, charge and mass of macro-ion number $i$, but here the charge and mass are equal to the physical values multiplied by a statistical weight equal to the number of physical particles represented by this macroparticle. A macro-ion of a given species has the same charge-to-mass ratio as the physical particles of this species.

When the electron inertia can be ignored, as is the case in this study, a massless electron fluid enforces instantaneous charge neutrality of the plasma: meanwhile, it exerts a pressure on the ions via the self-consistent electric field and contributes to the total current density involved in Ampère's equation. The electric field is determined by the balance of forces exerted on the unit volume of the electron fluid:

$0=-q n_{e}\left(\boldsymbol{E}+\boldsymbol{v}_{e} \times \boldsymbol{B}\right)-\nabla P_{e}$,

where $n_{e}, m_{e}, \boldsymbol{v}_{\boldsymbol{e}}$ and $P_{e}$ are, respectively, the electron density, velocity and pressure. The restriction to low frequency phenomena allows oneto neglect the displacement current in Ampère's equation and to obtain the electric field as a state function. Neglecting the electron inertia restricts the validity of the model to spatial scales greater than the electronic inertial length. The electronic temperature is determined by the equation of state, $P_{e}=n_{e} k_{B} T_{e}$, and the electronic pressure by assuming that the motion of the electron fluid is adiabatic with a polytropic index equal to 2 . This closure equation is valid as long as the electron $\beta$ is smaller than unity (Thomas, 1989).

The time evolution of the magnetic induction is governed by Faraday's equation, constrained by the conservation of the magnetic flux. The simulation model makes use of the algorithm developed by Matthews (1994) which allows an efficient computing of multi-species plasmas. An explicit presentation of the algorithm and validation tests is made by Matthews (1994).

The coordinate system is defined as follows: the $X$ direction is aligned with the solar wind bulk flow velocity $\boldsymbol{V}_{s w}$ and points away from the Sun, $Y$ is the direction of the motional electric field of the solar wind $\boldsymbol{E}_{s w}=-\boldsymbol{V}_{s w} \times \boldsymbol{B}_{I M F}$, where $\boldsymbol{B}_{I M F}$ is the Interplanetary Magnetic Field (IMF) conveyed by the solar wind, and the $Z$ direction is taken to complete a right-handed frame of reference. The cone angle between $\boldsymbol{V}_{s w}$ and $\boldsymbol{B}_{I M F}$ is an input parameter, which is equal to $90^{\circ}$ 
in runs discussed in this article. Simulations are computed on a 3-D uniform Cartesian grid with cubic cells having a size of $300 \mathrm{~km}$, which corresponds to twice the proton inertial length in the solar wind. The size of the simulation domain is defined by $-2.85 \leq X \leq+2.85$ Martian radii $\left(R_{M}\right)$ and $-6.6 \leq Y, Z \leq+6.6 R_{M}$. The obstacle is modelled by a fully absorbing sphere with a radius of $3400 \mathrm{~km}$ : the ions are stopped when they penetrate the obstacle. Let us notice that the ion dynamics below the exobase is not correct in our model because it does not take into account the friction with the collisional atmosphere, but this is not so important with the present spatial resolution of $300 \mathrm{~km}$ which is comparable to the altitude of the exobase; the dense atmosphere is thus represented by a transition layer between the collionless domain above the exobase and the solid planetary core. The present hybrid model ignores the crustal magnetic field of Mars and the basic ionospheric chemistry, successively included by Liu et al. (1999); Liu et al. (2001) and Ma et al. (2002); Ma et al. (2004). These points will be reconsidered in future works with improved resolution.

Open boundary conditions are taken for the entry and exit faces of the solar wind, and periodic boundary conditions are used in the $Y$ and $Z$ directions, except for pickup ions which are escaping freely from the simulation domain. No pickup ions are injected into the simulation through its boundary: they are created self-consistently inside the box (see Sect. 2.4). The code is run from time $t=0$ to time $t \simeq 1000 \mathrm{~s}:$ a nearly stationary regime is reached around time $t=470 \mathrm{~s}$

\subsection{Plasma description}

The model includes $\mathrm{H}^{+}$and $\mathrm{He}^{++}$solar wind ions with parameters corresponding to the solar wind parameters measured near the Martian orbit. Although the density of alpha particles is smaller than the proton density, they carry out a significant fraction of the solar wind mass and momentum ( $20 \%$ ), and thus can influence the overall dynamics. In order to eliminate statistical problems, we use the same number of macroparticles per cell to represent both ion species and adjust the statistical weights of the macroparticles to render the physical composition of the solar wind plasma. The hybrid code used for this study is robust enough to be run with small numbers of macroparticles per cell, as long as the level of fluctuations is compatible with the physical processes investigated, with the main drawback being a loss of accuracy in the energy balance. Since the region upstream of the bow shock is not the target of this study, it is represented by as few macroparticles as possible, i.e. 2 macro-protons and 2 macro-alphas per cell, on average. The protons number density is chosen equal to $2.3 \mathrm{~cm}^{-3}$ and the undisturbed velocity distribution functions of solar wind ions are assumed to be Maxwellian, with a common bulk velocity of $400 \mathrm{~km} \mathrm{~s}^{-1}$ for both species. The interplanetary magnetic field strength is $3 \mathrm{nT}$. With these parameters, the Alfvén and sonic speeds are about $40 \mathrm{~km} \mathrm{~s}^{-1}$, indicating a highly supersonic and super-Alfvénic solar wind with sonic and Alfvén Mach numbers close to 10.

Planetary ions come either from an ionospheric source or are produced in-situ by the ionization of the neutral exospheric constituants. Ionization processes creating $\mathrm{H}^{+}$and $\mathrm{O}^{+}$ions will be discussed in more detail in Sect. 2.4. The ionospheric source is modeled by the creation of $\mathrm{O}^{+}$and $\mathrm{O}_{2}^{+}$ ions uniformly implanted in the altitude range from 250 to $350 \mathrm{~km}$. The ionosphere is supposed to inject ions at a rate corresponding to an ionospheric flux escape of $10^{7} \mathrm{~cm}^{-2} \mathrm{~s}^{-1}$ on the dayside, and ten times less on the nightside (Liu et al., 1999).

\subsection{The neutral environment}

In our model, the Martian neutral environment is modelled by two coronae of atomic hydrogen and oxygen having spherical symmetry. Furthermore, the radial profile of density for each source depends upon the level of the solar EUV flux. This simplified approach neglects the dependency of the exospheric densities upon the local time and latitude. Three-dimensional Monte-Carlo simulations of the hot oxygen corona made by Hodges (2000) demonstrate a significant day-night asymmetry, with the exosphere being about three times denser on the dayside than on the nightside. These simulations also demonstrate a weak influence of the planetary rotation, resulting in a small dawn-dusk dissymmetry of the hot atomic oxygen corona. The important day-night asymmetry, presently not taken into account in our model, will be included in our future studies.

The radial variation of the number density of atomic hydrogen is given by a simplified Chamberlain model corresponding to an isothermal equilibrium in the gravity field of the planet:

$$
\begin{aligned}
n_{H}(h) & =n_{H}\left(h_{0}\right) \exp \left(H_{0}\left(\frac{1}{R_{M}+h}-\frac{1}{R_{M}+h_{0}}\right)\right) \\
H_{0} & =\frac{G M_{M} m_{H}}{k T_{H}},
\end{aligned}
$$

where $n_{H}\left(h_{0}\right)$ is the neutral hydrogen density at the exobase, $h_{0}$ is the altitude of the exobase, $G$ the gravitational constant, $M_{M}$ the mass of Mars, $m_{H}$ the atomic mass of hydrogen, and $T_{H}$, the temperature of hydrogen at the exobase. The density and temperature at the exobase are the input parameters which control the extension of the hydrogen corona: For this study we have adopted values given by Krasnopolsky $(1993 a, b)$ to characterize conditions at solar minimum and solar maximum. For the oxygen atomic corona we chose the radial profile computed by Kim et al. (1998) by means of Monte-Carlo simulations; it includes both hot and cold oxygen populations. The main source of hot oxygen atoms is the dissociative recombination of the molecular ions $\mathrm{O}_{2}^{+}$ which occurs through a few different channels characterized by a different branching ratio and different energies of the released atoms.

Figure 1 shows the density profiles for atomic oxygen and hydrogen as a function of the radial distance from 


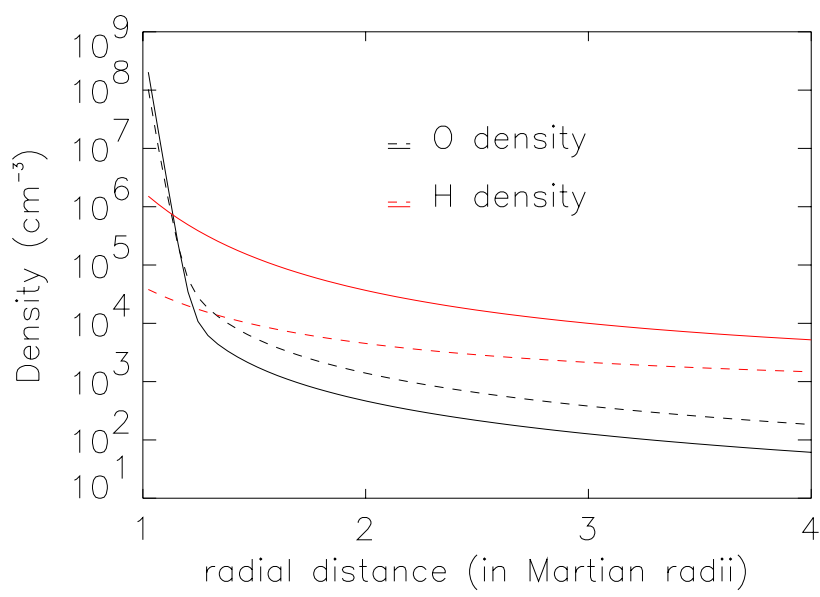

Fig. 1. Neutral hydrogen and oxygen coronae at solar minimum and solar maximum. Solid curves represent the densities during solar minimum while dashed curves correspond to the solar maximum conditions.

Mars' centre; it gives clear evidence of the large variations occuring between the solar minimum and maximum. Let us notice that densities reached in the region upstream of the bow shock, i.e. at distances $\left(\geq 1.5 R_{M}\right)$, are significant. That allows a substantial production of planetary ions with a corresponding loss of atmospheric neutrals.

\subsection{Ionization processes}

Planetary ions are produced by three processes: photoionization, electron impacts and charge exchanges. We propose a new approach contrasting with previously published hybrid simulations: the global ion production is not an input parameter of our model but is calculated self-consistently. The local production rate of each ionic species is computed from the assumed neutral reservoirs and the self-consistent dynamics of the ions by using model cross sections or ionization frequencies: this approach allows one to distinguish contributions from the different mechanisms and to provide independent estimations of the global production of various ionic species which can be compared to extrapolations made from local spacecraft measurements.

Solar EUV photons can produce $\mathrm{H}^{+}$and $\mathrm{O}^{+}$ions by the following reactions:

$h v+\mathrm{O} \longrightarrow \mathrm{O}_{p l}^{+}+e^{-}$

$h v+\mathrm{H} \longrightarrow \mathrm{H}_{p l}^{+}+e^{-}$,

where the subsript $p l$ refers to planetary ions. Since the photoionization frequencies depend on the ionizing photon flux, which varies with the phase of the solar cycle, we adopt different values for solar minimum and solar maximum conditions, as given in Table 1. Of course, there is no photoionization in the shadow of the planet.

The electron impact process is also included in our model,

$e^{-}+\mathrm{O} \longrightarrow \mathrm{O}_{p l}^{+}+2 e^{-}$
Table 1. Photoionization frequencies near Mars orbit for oxygen and hydrogen atoms as a function of the solar activity.

\begin{tabular}{rrrr}
\hline & & Solar min. & Solar max \\
\hline$f_{\mathrm{O}}$ & $\left(s^{-1}\right)$ & $10.7 \times 10^{-8}$ & $31.25 \times 10^{-8}$ \\
$f_{\mathrm{H}}$ & $\left(s^{-1}\right)$ & $2.40 \times 10^{-8}$ & $4.28 \times 10^{-8}$ \\
\hline
\end{tabular}

$e^{-}+\mathrm{H} \longrightarrow \mathrm{H}_{p l}^{+}+2 e^{-}$.

For an electron temperature of the free solar wind equal to $1.5 \times 10^{5} \mathrm{~K}$, the ionization rate of atomic oxygen per electron is estimated to be equal to $1.30 \times 10^{-8} \mathrm{~s}^{-1}$, while for atomic hydrogen it is estimated to be $0.76 \times 10^{-8} \mathrm{~s}^{-1}$. Assuming an adiabatic motion of the electron fluid with a polytropic index $\gamma=2$, the electron temperature varies as $T_{e} \propto n_{e}^{\gamma-1}$. Moreover the thermal energy of the electrons in the solar wind is close to the ionization thresholds of the considered species, which results an in ionization frequency per electron grossly proportional to the electron temperature, $v_{\text {impact }} \propto T_{e}$, according to published cross section (Kim et al., 2004); thus $v_{\text {impact }} \propto n_{e}^{2}$, where $n_{e}$ is the density of the solar wind electrons.

Charge exchange reactions are the third source of planetary ions. Hereafter, we call primary charge exchanges those occurring between a solar wind proton and a neutral atom, and secondary charge exchanges are the reactions between a planetary ion and a neutral atom. Reactions taken into account in our model are the following:

$$
\begin{array}{r}
\mathrm{H}_{p l, s w}^{+}+\mathrm{H} \longrightarrow \mathrm{H}^{E N A}+H^{+} \\
\mathrm{H}_{p l, s w}^{+}+\mathrm{O} \longrightarrow \mathrm{H}^{E N A}+\mathrm{O}^{+} \\
\mathrm{O}_{p l}^{+}+\mathrm{H} \longrightarrow \mathrm{O}^{E N A}+v^{+} \\
\mathrm{O}_{p l}^{+}+\mathrm{O} \longrightarrow \mathrm{O}^{E N A}+\mathrm{O}^{+},
\end{array}
$$

where subscripts mean solar wind $(s w)$ or Energetic Neutral Atom (ENA). We use model cross sections derived from results given by Stebbings et al. (1964). For nonresonant reactions $\left(\left(\mathrm{H}^{+}, \mathrm{O}\right)\right.$ and $\left.\left(\mathrm{O}^{+}, \mathrm{H}\right)\right)$ the cross sections are assumed to be constant while for resonant reactions $\left(\left(\mathrm{H}^{+}, \mathrm{H}\right)\right.$ and $\left.\left(\mathrm{O}^{+}, \mathrm{O}\right)\right)$ their dependency with respect to the relative velocity of the two particles is retained.

\section{Simulation results}

Solar EUV variations along the solar cycle modulate not only the photoionization frequencies, but also the densities of the neutral exospheric constituants (Fig. 1), as discussed in the preceding section. We will now discuss how these modulations affect the global pattern of the solar wind interaction with Mars. 


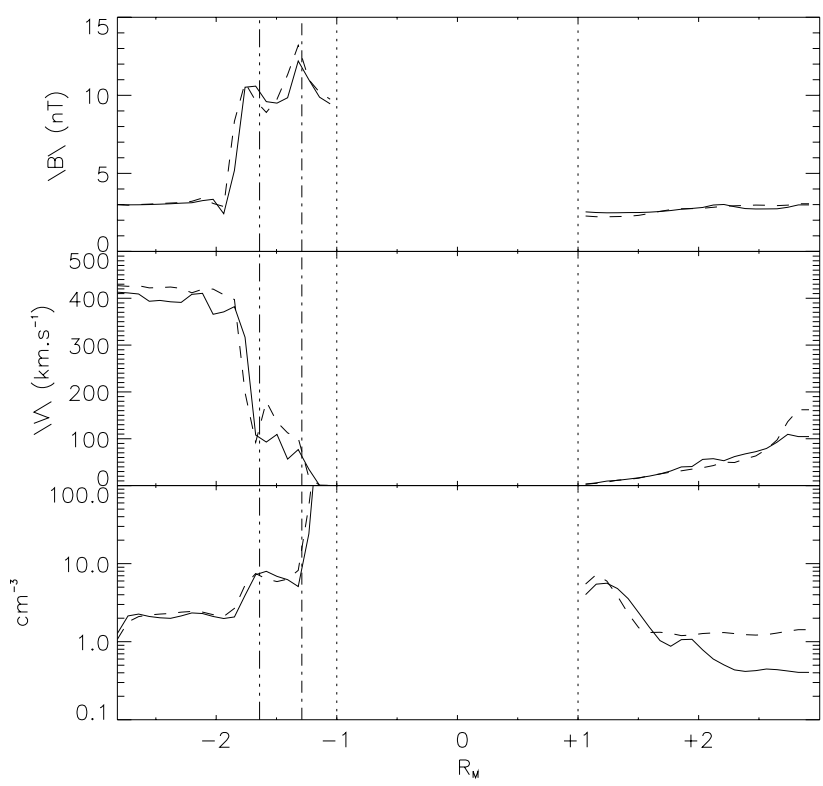

Fig. 2. Variations of the intensity of the magnetic field, of the bulk velocity, and of the total number density of the multi-species plasma along the Sun-Mars line. Values for solar minimum (maximum) conditions are shown by the solid (dashed) curves. The vertical dashed-dotted lines indicate the average subsolar positions of the bow shock (BS) and Magnetic Pile-up Boundary (MPB) deduced from the MGS observations.

\subsection{Global parameters}

Figure 2 shows the variations of the magnetic field strength (upper panel), of the bulk velocity (middle panel), and of the total number density (lower panel) of the multi-species plasma along the Sun-Mars line for conditions at solar minimum and at solar maximum. The Sun is on the left side and the solar wind flow goes to the right: the planet is delimited by the two vertical dotted lines. This figure clearly shows concomitant steep variations in the three panels around $R \simeq-1.7$ : the magnetic field intensity and the plasma number density, which is equal to the electron number density because the model involves only singularly charged ions, increase by a factor of $\simeq 4$ meanwhile, the bulk velocity of the solar wind decreases in the same proportion. These variations are clear signatures of the bow shock. The second steep increase of the plasma number density around $R \simeq 1.3$, concomitant with the final drop to zero of the bulk velocity of the plasma, corresponds to the maximal value reached by the magnetic field intensity in the simulation domain. This boundary is clearly separated from the obstacle, despite the coarse resolution of the simulation, and is clearly associated with the limit of the domain dominated by the planetary plasma, hence it seems legitimate to consider it as a simulated MPB. It can also be considered as the boundary of the induced Martian magnetosphere: the magnetic field intensity increases as the incident magnetic flux convected by the solar wind piles up against the planetary plasma and as the solar wind plasma becomes almost stagnant. On the dayside, three regions can be easily distinguished: the upstream region where the solar wind plasma density fluctuates around $2.3 \mathrm{~cm}^{-3}$, the downstream region between the bow shock and the MPB and finally, the region below the MPB where planetary ions dominate (see also Fig. 4). Subsolar positions of the boundaries which separate these regions are at $-1.62 R_{M}$ and $-1.29 R_{M}$, respectively. The average subsolar positions of the bow shock and of the MPB derived from MGS observations by Vignes et al. (2000) are indicated by two vertical dashed-dotted lines for comparison. Subsolar positions of both boundaries in the simulations are in reasonable agreement with the average values derived from the observations.

Figure 2 also gives evidence that the positions of both the bow shock and the MPB location are not significantly affected by the level of the solar EUV flux, at least with the spatial resolution of the present simulations, a conclusion in agreement with previous statistical analyses of bow shock crossings (Vignes et al., 2000). Let us note that the drop in the bulk velocity at the MPB is also in good agreement with the observations made by the experiment ASPERA on board Phobos-2 (Lundin et al., 1989).

No essential difference can be noticed in the plasma number density profile between low and high solar EUV flux, except on the nightside where the plasma density at solar maximum is 6 times larger than during solar min conditions. This contrast is essentially due to the variation of the planetary ion production.

Thus, global plasma parameters do not change drastically with the solar EUV flux, in particular, the subsolar positions of the bow shock and MPB, are almost independent of this flux. The Martian case contrasts with the Venusian case for which the position of the bow shock is significantly modulated by the solar cycle (Zhang et al., 1990).

\subsection{Solar wind and planetary ions}

The main features of the three-dimensional distribution of the plasma around Mars can be discussed by examining twodimensional cuts of the simulation domain by the coordinate planes: plane $X Y$, containing the bulk velocity $\boldsymbol{V}_{s w}$ and the motional electric field $\boldsymbol{E}_{s w}$ of the solar wind, and plane $X Z$, containing $\boldsymbol{V}_{s w}$ and the interplanetary magnetic field $\boldsymbol{B}_{I M F}$.

Maps of the electromagnetic field and of the plasma parameters show an asymmetry between the half subdomains corresponding to positive and negative $Y$ coordinates. This asymmetry is a consequence of the action of the motional electric field of the incoming plasma on the planetary ions which are accelerated mainly along the direction of the convection electric field, i.e. in the positive $Y$ direction, during a fraction of their first gyroperiod. Since their gyroradii are of the order of the size of the Martian obstacle, this initial acceleration breaks the symmetry between the two sides of the planet.

Figure 3 shows an overview of the solar wind ion densities at the minimum solar EUV flux. Small and randomly 

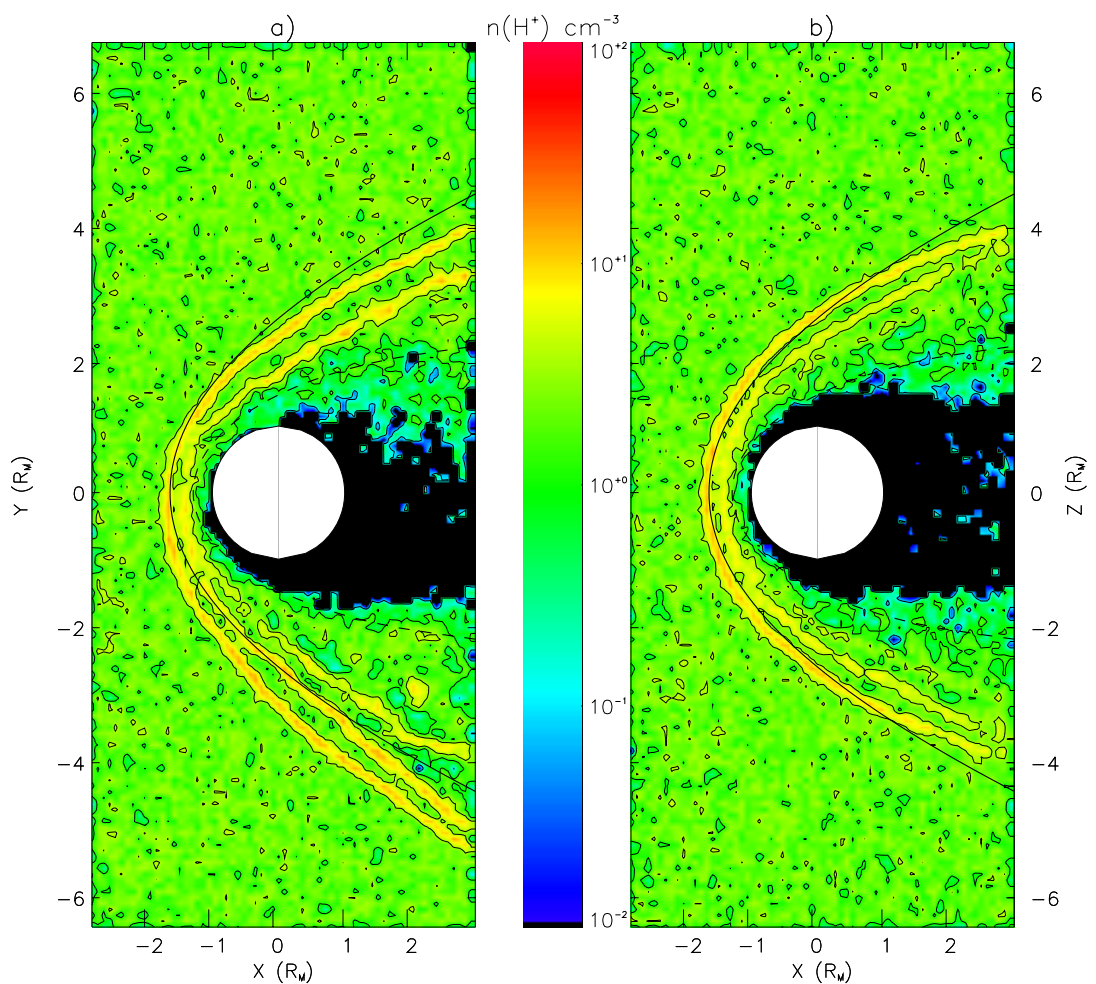

c)

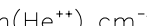

d)
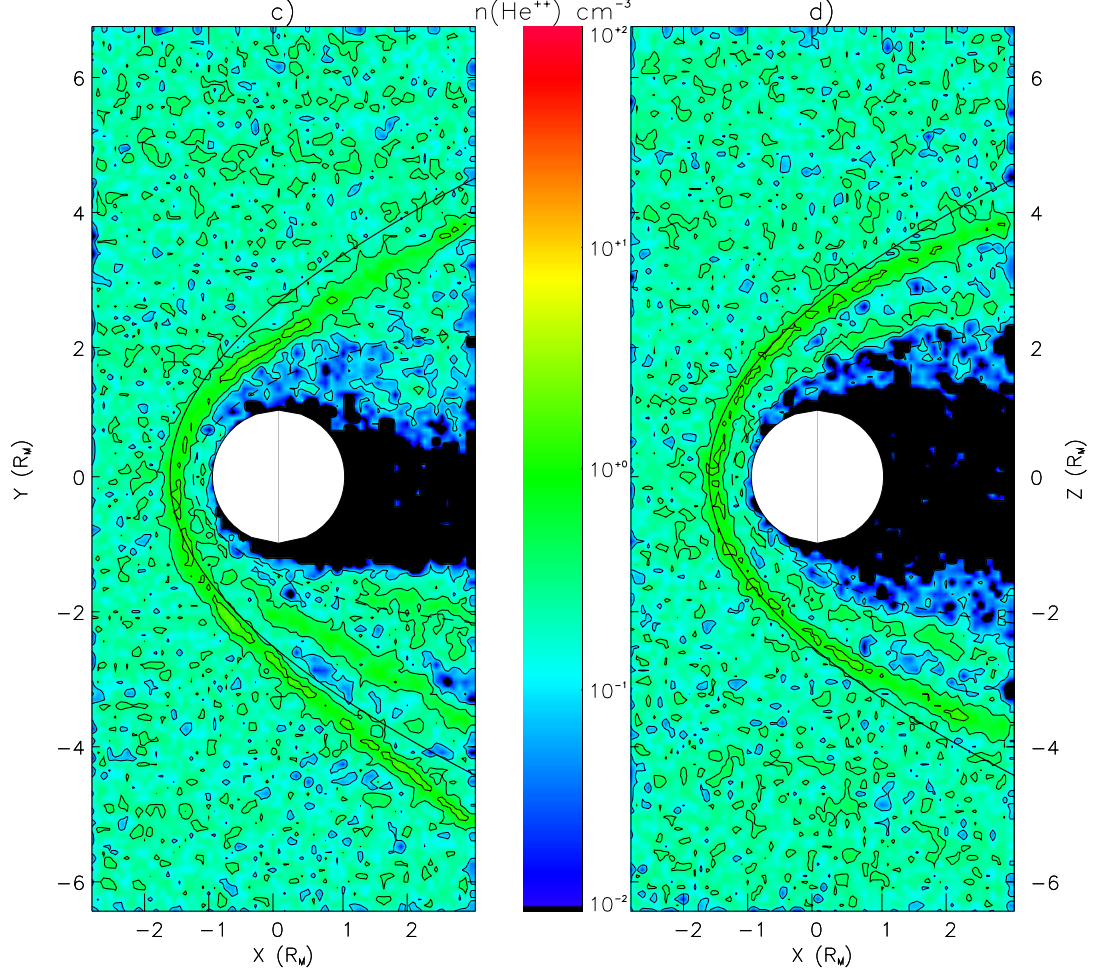

Fig. 3. Density maps of the solar wind ions. Top plates: Densities of the solar wind protons, left plate in the $X Y$ plane containing the motional electric field of the solar wind, right plate in the $X Z$ plane containing the IMF. Bottom plates: Densities of the solar wind alpha particles in the same planes. The number density of alpha particles in the unperturbed upstream solar wind is equal to 5\% of the proton number density. The solid and dashed black curves overlayed represent fits to the observations made by MGS, respectively, for the bow shock and the MPB (Vignes et al., 2000). 
distributed features are due to density fluctuations enhanced by rather small numbers of macroparticles per cell. All plates in Fig. 3 show the complex structure of the bow shock characterized by interlaced density enhancements: the number density of protons is maximal where the density of alpha particles is minimal and vice versa. The most upstream density enhancement is due to protons. The phase opposition between the oscillations of protons and alpha particles is remarkable and clearly in agreement with results of bi-ion fluid simulations by Sauer et al. (1996), who simulated by means of a two-dimensional bi-ion fluid code a plasma made of protons and $2 \%$ of heavy ions incident on a small obstacle: despite the fact that they used a ratio of ion masses equal to 20 , more adapted to pickup ions in a cometary environment, they reported a splitting of the bow shock quite similar to what is observed here with a solar wind made of protons and alpha particles. Results from hybrid simulations published by other authors (e.g. Shimazu, 2001) have been obtained with an incident solar wind made of protons only: nevertheless, they display a similar fine structure of the bow shock, but MHD simulated bow shocks never exhibit such a fine structure. This splitting of the bow shock could be due to dispersion originating either in kinetic effects when only one species is involved, as suggested by Shimazu (2001), or in biion effects suggested by Sauer et al. (1996). It is worth noticing that bi-ion effects can occur in a plasma characterized by two different populations of the same chemical species, for example, protons directly streaming through the BS and protons reflected at the shock front and transmitted downstream after they have gained energy. Thus, bi-ion effects cannot be discarded in explaining the fine structure of the bow shock observed in single species simulations, too. This issue still needs clarification: mechanisms leading to the formation of these structures have not yet been elucidated and require further comparative studies to be made with and without helium ions in the solar wind. Dubinin et al. $(1996,1998)$ have reported a similar sequence of alternating layers of protons and planetary oxygen ions observed by Phobos-2, but in the magnetosheath. They show that this plasma stratification leads to a change in the ion composition of the plasma from the magnetosheath dominated by protons to the planetary wake dominated by oxygen ions. We have not observed such oscillations near the composition boundary of the planetary wake in the simulations discussed here, possibly due to a lack of spatial resolution. Bi-ion effects could offer a common explanation for oscillations of composition observed at the bow shock and near the composition boundary, but more work is required to reach this conclusion.

Both Figs. 3a and c, presenting, respectively, the number densities of protons and alpha particles in the $X Y$ plane, clearly show an asymmetry of the bow shock which is not observed in the other cut by the plane $X Z$. In the present simulations, as in other hybrid simulations for Mars (Shimazu, 2001) and even for Venus (Moore et al., 1991), all are made with a constant IMF, where the bow shock occurs at larger distances from the planet on the side opposite to the convection electric field of the solar wind, which is also the side of the locally downward convective electric field. Vignes et al. (2002) in their study of the Martian bow shock crossing by MGS, concluded that "the shock appears significantly farther from the planet in the hemisphere of locally upward interplanetary magnetic field when the angle between the solar wind direction and the interplanetary magnetic field is large". The reason for this contradiction between the numerical simulations and the MGS data analysis is presently not understood and requires further investigation: perhaps the hypothesis of a spherically symmetric exosphere, common to all simulations, is questionable due to the effect of sputtering.

Another important feature evidenced by Fig. 3 is the cavity carved by the planetary obstacle in the solar wind plasma, a feature confirmed by Phobos-2 observations (Lundin et al., 1990b; Dubinin et al., 1996). It was observed that the plasma of Martian origin dominates the central tail region where protons are less abundant by a factor of 100 . Kallio and Janhunen (2001) have also described the formation of a proton cavity on the nightside. The results presented here show distinctly that a solar wind proton cavity is also observed on the dayside. However, the solar protons penetrate close to the planet in the equatorial local time sector between 09:00 and 12:00 LT, i.e. on the $y \geq 0$ side of the diurnal hemisphere. This effect has been previously reported by Kallio and Janhunen (2001) and Brecht (1997). A penetration of solar wind protons has been recently identified with the ASPERA-3 experiment on board Mars-Express spacecraft. Lundin et al. (2004) show that protons can penetrate down to an altitude of $250 \mathrm{~km}$. Due to their larger inertia, alpha particles penetrate closer to the planet than protons do. In the wake, the cavity is characterized by a boundary layer having a width ranging from $\simeq 0.1 R_{M}$ around the terminator on the $y \leq 0$ side in the $X Y$ plane, to $\simeq 1 R_{M}$ on the opposite side in the same plane, with intermediates and equal values in $X Z$ plane. Boundary layers of protons and alpha particles have comparable widths at a given location. In the $X Y$ plane the cavity is not centred on the Sun-Mars line but is shifted by $\simeq 0.5 R_{M}$ in the $y \leq 0$ direction, opposite to $\boldsymbol{E}_{s w}$. The shift seems to be larger for the protons than for the alpha particles. In the $X Z$ plane, both cavities are symmetric with respect to the Sun-Mars line.

The variations of the number densities of different ion species along the Sun-Mars line are displayed in Fig. 4 for solar maximum (top panel) and solar minimum (bottom panel). The ion composition changes when approaching the planet: the densities of planetary ions, mainly formed by photoionization and charge exchanges, gradually increase until they become larger than the densities of solar wind ions. A similar transition has been observed by the ASPERA experiment on board the Phobos-2 spacecraft (Lundin et al., 1990b; Dubinin et al., 1996) and in bi-ion fluid simulations (Sauer and Dubinin, 2000). On the dayside, the MPB corresponds roughly to the boundary that separates the region dominated by solar wind ions from the region where planetary ions dominate. It is seen that $\mathrm{O}^{+}$ions largely dominate below $1000 \mathrm{~km}$, as observed by ASPERA on board Phobos-2 (Lundin et al., 1991). Traces of planetary protons are visible at altitudes 


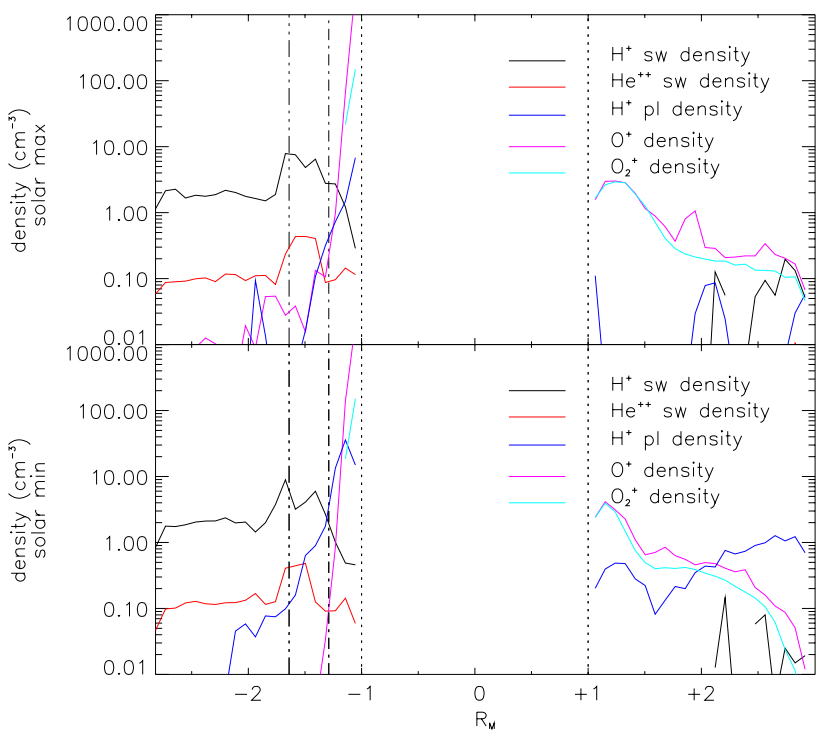

Fig. 4. Ions density profile following the Sun-Mars direction. Planetary $\left(\mathrm{H}_{p l}^{+}, \mathrm{O}^{+}\right.$and $\left.\mathrm{O}_{2}^{+}\right)$and solar wind ions $\left(\mathrm{H}_{s w}^{+}\right.$and $\left.\mathrm{He}^{++}\right)$densities are plotted in both cases: solar maximum (top panel) and solar minimum (bottom panel). The first vertical dashed-dotted line represents the average subsolar point of the bow shock, and the second vertical dashed-dotted line represents the subsolar point of the MPB.

greater than 2-Martian radii in Fig. 4 (see also Fig. 5). These ions, only protons at solar minimum, picked up by the solar wind, have also been observed by the plasma spectrometer ASPERA on board the Phobos-2 spacecraft (Barabash et al., 1991).

Solar wind ions are absent on the nightside, and the main constituents are planetary ions of ionospheric or exospheric origin. Close to the planet, $\mathrm{O}^{+}$and $\mathrm{O}_{2}^{+}$densities reach $4 \mathrm{~cm}^{-3}$, while at 2-Martian radii these densities are still greater than $0.1 \mathrm{~cm}^{-3}$ at solar maximum. These simulated densities are comparable with Phobos- 2 observations in the tail region where the $\mathrm{O}^{+}$density reaches $6 \mathrm{~cm}^{-3}$ (Lundin et al., 1989, 1990a). The difference between the densities of $\mathrm{O}^{+}$ions at the solar minimum and maximum can be explained by the fact that the oxygen neutral corona is denser and more extended at solar maximum. This contrasts with the behaviour of the hydrogen corona which contracts at solar maximum, thus decreasing the production of planetary protons. These features are clearly displayed in Fig. 5, which describes the behaviour of the planetary ions at solar maximum and at solar minimum. At solar maximum there are no planetary protons upstream of the bow shock and a steep increase in their density is clearly seen close to the observed average MPB. As shown by plate a) their spatial distribution below the MPB is strongly asymmetric: their number density varies from $\simeq 1 \mathrm{~cm}^{-3}$ on the $y \leq 0$ side, to less than $10^{-2} \mathrm{~cm}^{-3}$ on the $y \geq 0$ side in the near wake. Close to the planet, maximal densities are observed in the local time sector between 09:00 and 12:00 LT, i.e. in the $+\boldsymbol{E}_{s w}$ hemisphere, while their penetration to the nightside cavity is more efficient in the opposite hemisphere, the $y \leq 0$ side. Plate b) clearly demonstrates the existence of ray-like structures stretching towards the tail in the $X Z$ plane containing the IMF. At solar minimum the hydrogen neutral corona inflates, and the distribution of planetary protons becomes more uniform in the wake, with the $(y \leq 0)$ side remaining more populated than the $y \geq 0$ side. A significant increase in the density of the planetary protons is noticed in the sheath between the BS and the MPB and even upstream of the bow shock (see also Fig. 4).

Figure $5 \mathrm{c}$ demonstrates a strong asymmetry of the distribution of oxygen ions in the $X Y$ plane containing $\boldsymbol{E}_{s w}$. At solar maximum, when the corona of atomic oxygen is enhanced, $\mathrm{O}^{+}$ions are recorded even upstream of the BS: their density on the $y \geq 0$ side is greater than $10^{-2} \mathrm{~cm}^{-3}$, creating what could be called an "oxygen foreshock". The maximal density in this region forms a curved ridge originating from the subsolar region of the BS and delineating the cycloidal motion of pickup oxygen ions created there. At solar minimum the most salient features are a drastic reduction in the upstream population of oxygen ions and an increased asymmetry in the wake, where the $y \geq 0$ side is more dense than the $y \leq 0$ side: the opposite of the observation made for the protons. Such observations have also been reported by Kallio and Janhunen (2002). This asymmetry of the oxygen distribution has also been noticed in test particles simulations (Kallio and Koskinen, 1999; Lichtenegger and Dubinin, 1998). Figures 5d and $\mathrm{h}$ offer another illustration, in the $X Z$ plane, of the drop in the oxygen ion density due to the shrinking of the atomic oxygen corona at solar minimum. But the essential feature demonstrated here is the confinement of the oxygen ions close to the equator, between the magnetic lobes formed by the draping of the IMF over the planetary obstacle. This plasma sheet is formed by ions extracted from the nightside ionosphere: its density varies by a factor of 4 between solar minimum and solar maximum, whereas the upstream density of oxygen ions varies by more than a factor of 10 in the upstream region (see Figs. $5 g$ and $h$ ).

\section{Estimation of the ionic escape}

Due to the absence of a global intrinsic magnetic field, the Martian atmosphere is exposed to a continuous erosion process because the ionized component can be efficiently removed by ion pickup mechanisms. On the basis of the Phobos- 2 observations it was estimated that the total escape of the planetary matter at solar maximum can reach $\sim 1 \mathrm{~kg} / \mathrm{s}$ (Lundin et al., 1990a). However, these estimates were made by using the in-situ plasma measurements and by assuming a cylindrical symmetry in the distribution of the outflowing ions. On the other hand, the simulations clearly show a strong asymmetry of the ion flows near Mars. Therefore three-dimensional hybrid simulations can help for estimating the loss of matter. Table 2 gives escaping fluxes of the different ionic species considered in our model: a benefit of the 

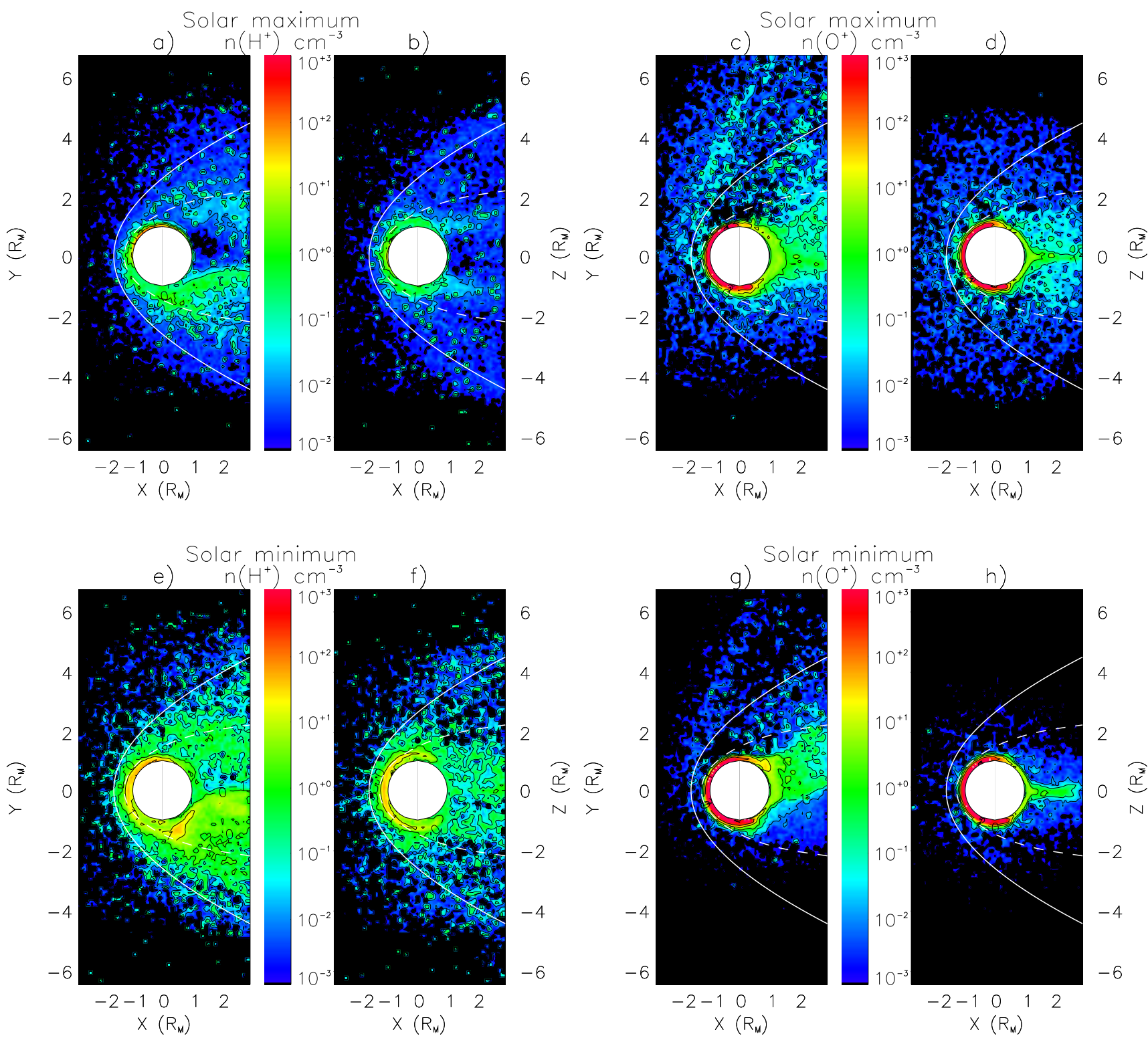

Fig. 5. Planetary ions. Density maps of planetary ions at solar maximum are presented on plates (a to d), and on plates (e to f) at solar minimum. Plates (a), (b), (e), and (f), refer to planetary protons while oxygen ions are displayed on plates (c, d, g), and (h). Plates (a), (c), (e), and (g) present density maps in the $X Y$ plane containing the convection electric field of the solar wind, whereas plates (b), (d), (f), and (h) display density maps in the $X Z$ plane containing the IMF. Solid and dashed white curves are the fits of MGS observations for the BS and the MPB made by Vignes et al. (2000). 
Table 2. Computed fluxes of escaping ions from three-dimensional hybrid simulation for maximum and minimum solar conditions. Contributions of various ionization are detailed following the ionization origin of the planetary ions.

\begin{tabular}{lcc}
\hline & \multicolumn{2}{c}{ Escaping Flux $\left(\times 10^{24}\right.$ ions.s $\left.^{-1}\right)$} \\
\cline { 2 - 3 } & Solar min & Solar max \\
\hline$O^{+}$photoionization & 0.29 & 1.68 \\
$H^{+}$photoionization & 3.52 & 1.16 \\
\hline$O^{+}$electron imp. ion. & 0.03 & 0.09 \\
$H^{+}$electron imp. ion. & 1.62 & 0.31 \\
\hline$O^{+}$charge exchange & 0.18 & 0.54 \\
$H^{+}$charge exchange & 37.7 & 9.93 \\
\hline $\mathrm{O}^{+}$ionosp. esc. & 0.04 & 0.06 \\
$\mathrm{O}_{2}^{+}$ionosp. esc. & 0.05 & 0.07 \\
\hline $\mathrm{H}^{+}$total & 42.8 & 11.4 \\
$\mathrm{O}^{+}$total & 0.52 & 2.37 \\
$\mathrm{O}_{2}^{+}$total & 0.05 & 0.07 \\
\hline
\end{tabular}

new approach adopted here to model the ionization processes (see Sect. 2.4) is the possibility to estimate separately the contributions from the various mechanisms. Table 2 clearly shows that the oxygen escape, as well as the hydrogen escape, is strongly dependent on the solar EUV flux. Whatever the solar EUV flux is, between 85 and $90 \%$ of the escaping proton flux comes from charge exchange. The escape flux of $\mathrm{H}^{+}$ions is approximately four times higher during solar minimum than during solar maximum, due to the inflation of the neutral hydrogen corona at solar minimum. On the other hand, photoionization is the main process which contributes to the escape of $\mathrm{O}^{+}$ions, which maximizes at solar maximum with the extension of oxygen corona. The loss rate of oxygen ions is about ten times smaller than the value given by Lundin et al. (1990a) from the ASPERA measurements $\left(\simeq 2.5 \times 10^{25}\right.$ ions/s) and is in better agreement with the estimates by Verigin et al. (1991) equal to $5 \times 10^{24}$ ions/s from the TAUS measurements on board Phobos-2. The different assumptions made about the distribution of ion fluxes in the Martian tail explain this discrepancy between experimentals estimations.

\section{Conclusions}

A full three-dimensional multi-species hybrid model has been used to characterize the Martian plasma environment and to estimate the escape of planetary ions during solar maximum and solar minimum. A self-consistent calculation of the global ion production is implemented in our model, leading to an independent estimation of the global production. The full dynamic of four ions species $\left(\mathrm{H}^{+}, \mathrm{He}^{++}, \mathrm{O}^{+}\right.$and $\mathrm{O}_{2}^{+}$) is described in our model. The contribution of alpha particles to the solar wind dynamic is included, for the first time, in hybrid simulations.

The solar EUV flux governs photoionization frequencies, as well as neutral densities of the Martian environment. We found that the solar flux does not drastically change global parameters such as magnetic field, bulk velocity and total density. In particular, the fact that subsolar positions of the bow shock and the MPB are independent of the solar EUV flux does not contradict observations. A strong asymmetry with respect to the direction of the convective electric field is revealed by the distribution of the plasma around the planet: solar wind ions penetrate closer to the planet in the equatorial plane containing the motional electric field of the solar wind, and oxygen ions are found upstream of the bow shock far away from the planet and on its $y \geq 0$ side. The complex structure of the bow shock is emphasised by interlaced solar wind ions densities, where the maximum proton density corresponds to minimum alpha particle density and vice versa. Moreover, a cavity void of solar wind ions is clearly identified. This cavity, filled by planetary ions, extends rather on the nightside than on the dayside and exists at both solar maximum and solar minimum.

The presented results clearly indicate a transition from the solar wind dominated plasma to the planetary plasma which roughly corresponds to the Magnetic Pile-up Boundary identified by MGS. A major outcome of these simulations is the prediction of the spatial distribution of planetary ions species: planetary protons populate almost uniformly the wake, whereas oxygen ions seem to be essentially present between these two magnetic lobes, in the plasma sheet. The evaluation of the oxygen loss rate at solar maximum, computed by our simulation model $\left(\sim 2.4 \times 10^{24}\right.$ ions/s) is less than estimated values from the Phobos- 2 mission. The escaping flux of oxygen ions is larger during solar maximum, contrary to the hydrogen flux which is larger at solar minimum.

Overall, the analysis shows the importance of kinetic effects in the solar wind interaction with the Martian neutral environment.

Acknowledgements. The authors wish to thank the "Institut du Développement et des Ressources en Informatique Scientifique" (IDRIS, Orsay, France) for the access to supercomputing facilities provided through project number 040294 "Modélisation et simulation numérique des plasmas planétaires". The authors would also like to express their aknowlodgements to the reviewers for their helpful comments which contributed to clarify and improve this article. One of us (G. M. C.) is grateful to K. Sauer at Max Planck Institute for Solar System Research in Lindau, Germany, for fruitful discussions during two invited stays in 2001 and 2003.

Topical Editor T. Pulkkinen thanks C. Mazelle and another referee for their help in evaluating this paper.

\section{References}

Acuña, M. H., Connerney, J. E. P., Wasilewski, P., Lin, R. P., Mitchell, D., Anderson, K. A., Carlson, C. W., McFadden, J., Rème, H., Mazelle, C., Vignes, D., Bauer, S. J., Cloutier, P., and 
Ness, N. F.: Magnetic field of Mars: Summary of results from the aerobraking and mapping orbits, J. Geophys. Res., 106(E10), 23 403-23 418, 2001.

Acuña, M. H., Connerney, J. E. P., Wasilewski, P., Lin, R. P., Anderson, K. A., Carlson, C. W., McFadden, J., Curtis, D. W., Mitchell, D., Rème, H., Mazelle, C., Sauvaud, J. A., d’Uston, C., Cros, A., Medale, J. L., Bauer, S. J., Cloutier, P., Mayhew, M., Winterhalter, D., and Ness, N. F.: Magnetic field and plasma observations at Mars: Initial results of the Mars Global Surveyor, Science, 279, 1676-1680, 1998.

Barabash, S., Dubinin, E., Pisarenko, N., Lundin, R., and Russell, C. T.: Picked-up protons near Mars - PHOBOS observations, Geophys. Res. Lett., 18, 1805-1808, 1991.

Brecht, S. and Ferrante, J.: Global hybrid simulationof unmagnetizedd planets: comparison of Venus and Mars, J. Geophys. Res., 98, 17 251-17 262, 1993.

Brecht, S. H.: Solar wind proton deposition into the Martian atmosphere, J. Geophys. Res., 102, 11 287-11 294, 1997.

Bruno, R., Villante, U., and Stecca, A.: Selected solar wind parameters at $1 \mathrm{AU}$ through two solar activity cycles, Ann. Geophys., 12, 105-112, 1994,

\section{SRef-ID: 1432-0576/ag/1994-12-105.}

Dubinin, E., Sauer, K., Baumgärtel, K., and Srivastava, K.: Multiple shocks near Mars, Earth Planets and Space, 50, 279-287, 1998.

Dubinin, E. M., Sauer, K., Lundin, R., Baumgartel, K., and Bogdanov, A.: Structuring of the transition region (plasma mantle) of the Martian magnetosphere, Geophys. Res. Lett., 23, 785-788, 1996.

Hodges, R. R. J.: Distributions of hot oxygen for Venus and Mars, J. Geophys. Res., 105, 6971-6982, 2000.

Kallio, E. and Janhunen, P.: Atmospheric effects of proton precipitation in the martian atmosphere and its connection to the Marssolar wind interaction, J. Geophys. Res., 106, 5317-5634, 2001.

Kallio, E. and Janhunen, P.: Ion escape from Mars in a quasi-neutral hybrid model, J. Geophys. Res., 107, doi:10.1029/2001JA000090, 2002.

Kallio, E. and Koskinen, H.: A test particle simulation of the motion of oxygen ions and solar wind protons near Mars, J. Geophys. Res., 104, 557-580, 1999.

Kim, J., Nagy, A. F., Fox, J. L., and Cravens, T. E.: Solar cycle variability of hot oxygen atome at Mars, J. Geophys. Res., 103, 29339 - 29342, 1998.

Kim, Y. K., Irikura, K. K., Rudd, M. E., Ali, M. A., Stone, P. M., Coursey, J. S., Dragoset, R. A., Kishore, A. R., Olsen, K. J., Sansonetti, A. M., Wiersma, G. G., Zucker, D. S., and Zucker, M. A.: Electron-Impact Ionization Cross Section Database (version 3.0), available:http://physics.nist.gov/ionxsec (23 August 2004,), National Institute of Standards and Technology, Gaithersburg, MD, 2004.

Krasnopolsky, V. A.: Solar Cycle Variations of the Hydrogen Escape Rate and the CO Mixing Ratio on Mars, Icarus, 101, 33-41, 1993a.

Krasnopolsky, V. A.: Photochemistry of the Martian atmosphere (mean conditions), Icarus, 101, 313-332, 1993b.

Lichtenegger, H. and Dubinin, E.: Model calculations of the planetary ion distribution in the Martian tail, Earth Planets and Space, 50, 445-452, 1998.

Liu, Y., Nagy, A. F., Groth, C. P. T., Zeeuw, D. L. D., and Gombosi, T. I.: 3D Multi-fluid MHD Studies of the Solar Wind Interaction with Mars, Geophys. Res. Lett., 26, 2689-2692, 1999.

Liu, Y., Nagy, A. F., Gombosi, T. I., Dezeeuw, D. L., and Powell, K. G.: The solar wind interaction with Mars: results of three- dimensional three-species MHD studies, Adv. Space Res., 27, 1837-1846, 2001.

Lundin, R., Borg, H., Hultqvist, B., Zakharov, A., and Pellinen, R.: First measurements of the ionospheric plasma escape from Mars, Nature, 341, 609-612, 1989.

Lundin, R., Zakharov, A., Pellinen, R., Barabasj, S. W., Borg, H., Dubinin, E. M., Hultqvist, B., Koskinen, H., Liede, I., and Pissarenko, N.: ASPERA/Phobos measurements of the ion outflow from the Martian ionosphere, J. Geophys. Res., 17, 873-876, 1990a.

Lundin, R., Zakharov, A., Pellinen, R., Borg, H., Hultqvist, B., Pissarenko, N., Dubinin, E. M., Barabasj, S. W., Liede, I., and Koskinen, H.: Plasma composition measurements of the Martian magnetosphere morphology, Geophys. Res. Lett., 17, 877-880, $1990 \mathrm{~b}$.

Lundin, R., Norberg, O., Dubinin, E. M., Pisarenko, N., and Koskinen, H.: On the momentum transfer of the solar wind to the Martian topside ionosphere, Geophys. Res. Lett., 18, 1059-1062, 1991.

Lundin, R., Barabash, S., Andersson, H., Homström, M., Grigoriev, A., Yamauchi, M., Sauvaud, J.-A., Fedorov, A., Budnik, E., Thocaven, J.-J., Winingham, D., Frahm, R., Scherrer, J., Sharber, J., Asamura, K., Hayakawa, H., Coates, A., Linder, D. R., Curtis, C., Hsieh, K. C., Sandel, B. R., Grande, M., Carter, M., Reading, D. H., Koskinen, H., Kallio, E., Riihela, P., Schmidt, W., Säles, T., Kozyra, J., Krupp, N., Woch, J., Luhmann, J., McKennaLawler, S., Cerulli-Irelli, R., Orsini, S., Maggi, M., Mura, A., Millio, A., Roelof, E., Williams, D., Livi, S., Brandt, P., Wurz, P., and Bochsler, P.: Solar Wind - Induced Atmopsheric Erosion at Mars : First Results from APERA-3 on Mars Express, Science, 305, 1933-1936, 2004.

Ma, Y., Nagy, A. F., Hansen, K. C., Zeeuw, D. L. D., and Gombosi, T. I.: Three-dimensional multispecies MHD studies of solar wind interaction with Mars in the presence of crustal fields, J. Geophys. Res., 107, 1282, doi:10.1029/2002JA009293, 2002.

Ma, Y., Nagy, A. F., Sokolov, I. V., and Hansen, K. C.: Threedimensional multispecies, high spatial resolution MHD studies of solar wind interaction with Mars, J. Geophys. Res., 109, a07211, doi:10.1029/2003JA010367, 2004.

Matthews, A.: Current Advance Method and Cyclic Leapfrog for 2D Multispecies Hybrid Plasma Simulations, Journ. Comp. Phys., 112, 102-116, 1994.

Mazelle, C., Winterhalter, D., Sauer, K., Trotignon, J. G., Acuña, M. H., Baumgärtel, K., Bertucci, C., Brain, D. A., Brecht, S. H., Delva, M., Dubinin, E., Øieroset, M., and Slavin, J.: Bow Shock and Upstream Phenomena at Mars, Space Sci. Rev., 111, 115181, 2004.

Moore, K. R., Thomas, V. A., and McComas, D. J.: Global hybrid simulation of the solar wind interaction with the dayside of Venus, J. Geophys. Res., 96, 7779-7791, 1991.

Nagy, A. F., Winterhalter, D., Sauer, K., Cravens, T. E., Brecht, S., Mazelle, C., Crider, D., Kallio, E., Zakharov, A., Dubinin, E., Verigin, M., Kotova, G., Axford, W. I., Bertucci, C., and Trotignon, J. G.: The plasma Environment of Mars, Space Sci. Rev., 111, 33-114, 2004.

Sauer, K. and Dubinin, E.: The Nature of the Martian 'Obstacle Boundary', Advances in Space Research, 26, 1633-1637, 2000.

Sauer, K., Bogdanov, A., and Baumgärtel, K.: Evidence of an ion composition boundary (protonopause) in bi-ion fluid simulations of solar wind mass loading, Geophys. Res. Lett., 21, 2255-2258, 1994. 
Sauer, K., Dubinin, E., Baumgärtel, K., and Bogdanov, A.: Bow shock 'splitting' in bi-ion flows, Geophys. Res. Lett., 23, 3643 3646, 1996.

Shimazu, H.: Three-dimensional hybrid simulation of magnetized plasma flow around an obstacle, Earth Planets and Space, 51, 383-393, 1999.

Shimazu, H.: Three-dimensional hybrid simulation of solar wind interaction with unmagnetized planets, J. Geophys. Res., 106, 8333-8342, 2001.

Spreiter, J. R. and Stahara, S. S.: Computer modeling of solar wind interaction with Venus and Mars, Washington, D.C., American Geophysical Union Geophysical Monograph Series, 66, 345383, 1992.

Stebbings, R. F., Smith, A. C. H., and Ehrardt, H.: Charge transfer between Oxygen atoms and $\mathrm{O}^{+}$and $\mathrm{H}^{+}$ions, J. Geophys. Res., 69, 2349-2355, 1964.

Tanaka, T.: Configuration of the solar wind flow and Magnetic field around the planets with no magnetic field : Calculation by a new MHD simulation scheme, J. Geophys. Res., 17 251-17 262, 1993.

Thomas, V. A.: Dimensionality effects in hybrid simulations of high Mach number collisionless perpendicular shocks, J. Geophys. Res., 94, 12 009-12 014, 1989.
Verigin, M. I., Shutte, N. M., Galeev, A. A., Gringauz, K. I., Kotova, G. A., Remizov, A. P., Rosenbauer, H., Hemmerich, P., Livi, S., Richter, A. K., Apathy, I., Szego, K., Riedler, W., Schwingenschuh, K., Steller, M., and Yeroshenko, Y. G.: Ions of planetary origin in the Martian magnetosphere (Phobos 2/TAUS experiment), Planet. Space Sci., 39, 131-137, 1991.

Vignes, D., Mazelle, C., Rème, H., Acuña, M. H., Connnerney, J. E. P., Lin, R. P., Mitchell, D. L., Cloutier, P., Crider, D. H., and Ness, N. F.: The Solar Wind interaction with Mars: locations and shapes of the Bow Shock and the Magnetic Pile-up Boundary from the observations of the MAG/ER experiment onboard Mars Global Surveyor, Geophys. Res. Lett., 27, 49-52, 2000.

Vignes, D., Acuña, M. H., Connerney, J. E. P., Crider, D. H., Rème, H., and Mazelle, C.: Factors controlling the location of the Bow Shock at Mars, Geophys. Res. Lett., 29, 42-1, doi:10.1029/2001GL014513, 2002.

Zhang, T.-L., Luhmann, J. G., and Russell, C. T.: The solar cycle dependence of the location and shape of the Venus bow shock, J. Geophys. Res., 95, 14 961-14 967, 1990. 